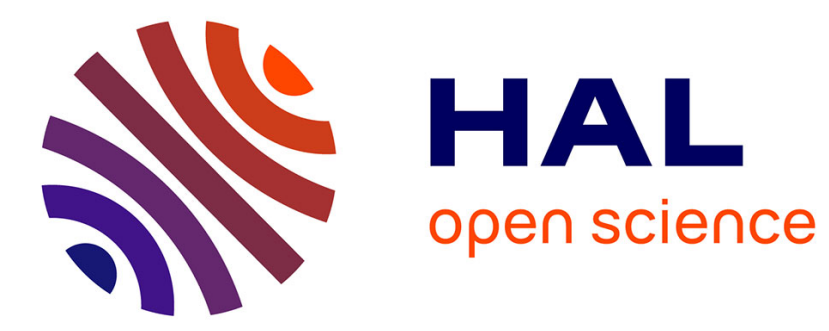

\title{
Indonésie La difficile instauration des réformes
}

Jean-Marc de Grave

\section{To cite this version:}

Jean-Marc de Grave. Indonésie La difficile instauration des réformes. L'Asie du Sud-Est 2016, pp.191219, 2016. halshs-03518233

\section{HAL Id: halshs-03518233 \\ https://shs.hal.science/halshs-03518233}

Submitted on 18 Jan 2022

HAL is a multi-disciplinary open access archive for the deposit and dissemination of scientific research documents, whether they are published or not. The documents may come from teaching and research institutions in France or abroad, or from public or private research centers.
L'archive ouverte pluridisciplinaire HAL, est destinée au dépôt et à la diffusion de documents scientifiques de niveau recherche, publiés ou non, émanant des établissements d'enseignement et de recherche français ou étrangers, des laboratoires publics ou privés. 


\section{L'Asie du Sud-Est 2016}

\section{Bilan, enjeux et perspectives}

\section{Sous la direction d'Abigaël Pesses}

Contributeurs :

Sarah Anaïs Andrieu, Jean Baffie, Julie Blot, François-Xavier Bonnet,

Bénédicte Brac de la Perrière, Christine Cabasset, Élodie Coffre, Barthélémy Courmont, Jean-Raphaël Chaponnière, Jean-Marc de Grave, Nathalie Fau, Éric Frécon, Laurent Gédéon, François Guillemot, Anne Yvonne Guillou, Bruno Jetin, Elsa Lafaye de Micheaux, Frédéric Lasserre, Éric Mottet, Abigaël Pesses et Marie-Sybille de Vienne

Avec la participation des stagiaires de l'Irasec, pour la chronologie par pays et le répertoire des principaux partis politiques :

Magali Lapouge, Nicolas Matthews et François Vezier

Mise en page, cartes et couverture : Bertrand Bayet Relecture orthographique : Alain Candille et Jeanne Lamie 
Les contributeurs

\section{Les dossiers de l'année}

L'Asean peut-elle transformer l'Asie du Sud-Est en région intégrée?

par Bruno Jetin

La Chine en Asie du Sud-Est : entre pré carré et rivalités géopolitiques grandissantes,

par Barthélémy Courmont, Frédéric Lasserre et Éric Mottet ....

L'Inde et l'Asie du Sud-Est, une relation proche mais lointaine,

par Jean-Raphaël Chaponnière

Les zones communes de développement : une solution en mer de Chine méridionale?

par Nathalie Fau

\section{Débat}

Appropriations politiques des rôles féminins en Asie du Sud-Est, débat entre Sarah Anaïs Andrieu, Élodie Coffre et Abigaël Pesses.

\section{La région}

Birmanie, par Bénédicte Brac de la Perrière

Brunei, par Marie-Sybille de Vienne

Cambodge, par Julie Blot et Anne Yvonne Guillou

Indonésie, par Jean-Marc de Grave.

Laos, par Éric Mottet.

Malaisie, par Elsa Lafaye de Micheaux

Philippines, par François-Xavier Bonnet

Singapour, par Éric Frécon

Thaillande, par Jean Baffie

Timor-Leste, par Christine Cabasset

Vietnam, par Laurent Gédéon et François Guillemot. 


\section{Indonésie La difficile instauration des réformes}

Jean-Marc de Grave

Durant cette première année de gouvernement, le président Joko Widodo - dit «Jokowi »- met en pratique les principales réformes qu'il a annoncées en matière de lutte contre la corruption, de mesures sociales ou de valorisation du domaine maritime. De ce point de vue, l'année écoulée se caractérise par un nombre d'avancées tout à fait significatives si l'on tient compte de l'ampleur du travail à accomplir.

Il reste que de telles mesures vont parfois à l'encontre de pratiques bien implantées, notamment celle de passe-droits liés au statut. Pour parvenir à imposer ses réformes, Jokowi doit donc jouer de compromis comme l'indiquent les reformulations d'alliances des partis politiques au sein du Parlement et les stratégies qui se mettent en place en vue des élections régionales de fin d'année 2015.

De plus, le résultat des élections présidentielles de 2014 illustre bien le fait que le paysage sociopolitique indonésien est en mutation. La période actuelle apparaît comme une phase transitoire où de nouvelles valeurs sociales et politiques se mettent en place, ce qui ne manque pas de provoquer des crises internes dans certains partis.

Les initiatives prises par le gouvernement permettent à Jokowi de conserver sa cote de popularité auprès de la classe moyenne. Mais l'augmentation du prix des produits de première nécessité mécontente les milieux économiquement défavorisés. Les critiques des opposants concernent d'ailleurs principalement l'économie, ce qui conduit à un renouvellement partiel du gouvernement durant la période estivale. 
Au point de vue international, l'accueil du nouveau président est plutôt bon, hormis le désaccord avec les gouvernements étrangers portant sur la condamnation à mort de certains de leurs ressortissants respectifs.

\section{Un paysage politique en devenir}

\section{Un nouveau gouvernement davantage ancré dans la société civile}

Suite aux élections présidentielles de juillet 2014, le nouveau gouvernement dont la constitution est annoncée le 26 octobre de la même année - revêt trois caractéristiques principales. Tout d'abord, ses membres sont jeunes : 20 des 34 ministres ont moins de 45 ans. Ensuite, ces derniers sont en majorité issus du monde professionnel : milieu des affaires - comme Joko Widodo - et milieu académique, principalement, soit là aussi 20 ministres sur 34, les autres provenant du monde politique ${ }^{313}$. Enfin, ils ont tous été soumis à l'appréciation de la Commission d'éradication de la corruption (KPK - Komisi Pemberantasan Korupsi) avant d'être retenus ${ }^{314}$.

Un aspect important de l'action du président est son ancrage dans les réseaux populaires, illustré par les groupes de «volontaires» qui ont soutenu le candidat Joko Widodo aux présidentielles et qui se voient hissés au statut $d^{\prime}$ «Organisations populaires » ${ }^{315}$. Mais ce succès ne peut suffire à asseoir sa légitimité sur la durée. L'entrepreneur apolitique qu'il est au départ doit tenir compte des contingences d'alliances politiques, justement, et des pratiques qui leur sont liées, en particulier lorsque le parti qui le soutient n'est pas majoritaire au Parlement et que les élections régionales sont proches.

\footnotetext{
${ }_{313}^{3}$ "Menghitung Jokowi Dalam Seratus Hari », Tempo, 8 février 2015, p. 50.

${ }^{314}$ Bruno Hellendorf, Enjeux d'une présidence lokowi. Réformes et relations civilo-militaires en Indonésie, Note d'analyse, Bruxelles, GRIP, 31 octobre 2014, p. 11, (http://www.grip.org/sites/grip.org/files/NOTES_ANALYSE/2014/NA_2014-10-31_FR_BHELLENDORFF.pdf).

315 "Sayap Barat Para Relawan ", Tempo, vol. 34, 26 octobre 2015, p. 56.
} 


\section{L'échéance des élections régionales}

Les élections régionales de décembre 2015 suscitent au long de l'année de nouveaux jeux d'alliance au sein du Parlement. Les enjeux se cristallisent notamment autour de la question du vote direct et indirect des représentants locaux : gouverneurs, préfets et maires. Le 2 octobre 2014, la question est votée en session plénière du Conseil des représentants du peuple (DPR), lequel correspond à la chambre haute du Parlement (MPR). Tout indique alors que si les élus locaux ne sont plus élus au suffrage direct, comme ils le sont depuis 2004, l'opposition deviendrait majoritaire dans les régions. Le Parti démocrate (PD), pour le maintien du vote direct et initialement allié sur ce point avec le Parti démocratique indonésien de lutte (PDI-P), le parti central de la coalition gouvernementale «Grande Indonésie » menée par Jokowi, effectue une volte-face. Ce retournement d'alliance permet ainsi à la coalition d'opposition «Rouge et Blanc », menée par Prabowo Subianto - candidat malheureux du second tour des présidentielles de 2014 - de remporter le vote à 226 voix contre $135^{316}$ pour rétablir le vote indirect.

L'événement révèle que le Parlement reste dominé par les pratiques d'enclicage contre lesquelles le nouveau gouvernement s'inscrit en faux ${ }^{317}$. L'accès au poste de président du DPR de Setya Novanto - cadre du Parti des groupes fonctionnels (Golkar - Partai Golongan Karya), parti central de la coalition «Rouge et Blanc »- l'illustre clairement ${ }^{318}$.

Quoi qu'il en soit, la préparation des régionales suit son cours. En juilletaoût 2015, la Commission des élections générales (KPU) distribue des tours de rôle aux villes et préfectures concernées pour permettre aux candidats de s'inscrire $^{319}$; en septembre, les candidats démarrent leur campagne ${ }^{320}$. Dans ce

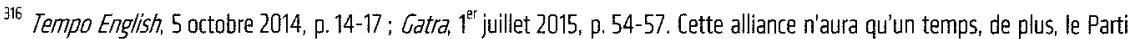
du mandat national (PAN - Partai Amanat Nasionai) quitte officiellement la coalition « Rouge et Blanc » début septembre 2015 dans le but de " soutenir le gouvernement"joko Widodo-jusuf Kalla ", ce qui fait gagner 49 sièges aux alliés du gouvernement, les rendant majoritaire au Conseil national (DPR) avec 295 sièges contre 204 ; "Pan Tinggalkan Koalisi Prabowo », Tempo, vol. 28,13 septembre 2015, p. 26.

${ }^{317}$ Tempo Eng/ish, 19 octobre 2014, p. $16-26$.

${ }^{318}$ «Tegakkan Martabat DPR », Tempo, vol. 36, 9 novembre 2014, p. 30 et 34-39.

319 " Komisi II DPR RI sepakat revisi UU Pilkada setelah 9 Desember », Antara News, 26 août 2015, (www.antaranews.com/ berita/514499/komisi-ii-dpr-ri-sepakat-revisi-uu-pilkada-setelah-9-desember).

320 "Kini bersekutu selepas berseteru », Tempo, août 2015, p. 28-30.
} 
processus, la crise interne que connaît le Golkar rend difficile pour lui une ligne cohérente de candidatures.

\section{Le Parti du Golkar en crise}

Le Golkar ${ }^{321}$ (91 sièges sur 560 au Parlement), le principal parti de l'opposition, est victime de querelles intestines qui le divisent en deux: les partisans d'Aburizal Bakrie, chef du parti depuis 2009, dont le mandat arrive à échéance, et ses opposants. En novembre 2014, suite à l'intervention de Jusuf Kalla (viceprésident d'Indonésie et président du Golkar de 2004 à 2009), Agung Laksono (vice-président du Golkar) est nommé chef du comité qui, pour rétablir l'ordre, démet Aburizal Bakrie de ses fonctions de chef du parti ${ }^{322}$.

L'origine du conflit remonte pour partie à la période de la campagne présidentielle, alors que le parti écarte Bakrie au profit d'une alliance avec Prabowo Subianto et Hatta Rajasa. En fin de compte, le cas est porté tout au long des mois de mars et avril devant différentes instances ministérielles, juridiques et constitutionnelles sans qu'un arrangement puisse être trouvé ${ }^{323}$, tant et si bien que des candidats locaux du Golkar s'inscrivent en juillet-août au nom des deux sections du parti pour être sûrs d'être retenus lorsque la décision des tribunaux aura été rendue.

La crise du Golkar révèle ainsi la faiblesse des partis lorsqu'ils ne sont pas menés par une personnalité qui fasse consensus. L'influence de Jusuf Kalla, devenu extérieur à l'organisation, ne peut suffire à combler cette absence. De plus, les pratiques internes des partis sont aussi tributaires des tensions extérieures qu'ils subissent. C'est notamment le cas de celles produites par la politique anticorruption du gouvernement.

\footnotetext{
${ }^{321}$ Même si le leader de l'« opposition » (la coalition « Rouge et Blanc »), Prabowo S., appartient à un autre parti (qu'il dirige), et alors que le vice-président, jusuf Kalla - bien qu'il soit du Golkar - a été choisi par jokowi lui-même.

${ }^{322}$ Agustina Widiarsi et al., "Beringin Terbelah Pisau Koalisi ", Tempo, 7 décernbre 2014, p. 34-35.

${ }^{323}$ Rohmat Haryadi et al, "Golkar Terbelah Semakin Parah », Gatra, vol, 22, 8 avril 2015, p. 10-21.
} 


\section{Résistance et compromis}

Une crise grave vient menacer la légitimité de la Commission d'éradication de la corruption, le KPK. Le trouble instauré suscite des réactions des institutions de la police, de la justice et du Conseil des représentants du peuple (DPR). L'événement indique que l'action du gouvernement est effectivement prise au sérieux par les partisans du système à l'ancienne, dit localement KKN (Korupsi, Kolusi, Nepotisme). Sur un plan plus général, le gouvernement doit également affronter la critique de l'affaiblissement économique du pays.

\section{Résistance des pratiques collusives}

Sous la pression de proches collaborateurs, Joko Widodo retient en janvier Budi Gunawan - officier de police controversé du fait de ses «comptes en banque obèses » - comme candidat au poste de chef de la police indonésienne, candidature acceptée par le DPR. Peu après, Gunawan est appelé à comparaître devant le KPK. Le chef de l'État revient alors sur sa décision. Considérant ce revirement comme un acte manqué, des officiers du bureau central de police lancent alors en guise de représailles une série d'accusations et de mises en demeure de comparution s'adressant aux membres de la Commission anticorruption $^{324}$. Le vice-président du KPK, Bambang Widjojanto, est ainsi assigné à comparaître dans une affaire de faux témoignage, puis arrêté le 23 janvier. Dans le même temps, trois autres dirigeants sont aussi convoqués ${ }^{325}$. Selon différents observateurs, ces incriminations seraient pour la plupart fictives ${ }^{326}$.

Ce travail de harcèlement se poursuit, tant et si bien qu'un comité de gestion de crise est constitué, qui décide de soumettre le cas de Gunawan à la Cour de justice $^{327}$. Après l'étude du dossier en procédure de préjugement celuici est déclaré «non suspect» par manque de preuves ${ }^{328}$. Cette procédure de préjugement affaiblit de fait l'autorité du KPK vis-à-vis, non seulement de la police, mais aussi de la Cour de justice, en ce qu'elle constitue un précédent à

\footnotetext{
${ }^{324}$ « Stop Kriminalisasi Pimpinan KPK », Tempo, voł, 49, 8 février 2015, p. 25.

${ }^{325}$ Dian Trivuli Handoko, "Peluru-peluru Membunuh Cicak », Tempo, vol. 49, 8 février 2015, p. 33.

325 «Kriminalisasi, Fitnah, Teror», Tempo, vol. 1, 8 mars 2015, p. 27; Rusman Paraqbueq et al, "Ancaman Bedil dari Trunojoyo ", Tempo, vol. 16, 21 juin 2015, p. 31-33.

${ }^{327}$ "Setelah Komisi Menyerah Kalah », Tempo, vol. 2, 15 mars 2015, p. 28-29.

${ }^{328}$ Sunudyantoro et al, "Dalam Tekanan Delapan Penjuru Mata Angin ", Tempo, vol. 14, 8 mars 2015, p. 8 ; Yuliawati et al, "Salah Asumsi, Salah Strategi », Tempo, vol. 12, 24 mai 2015, p. 90-91.
} 
d'autres cas retenus par la Commission anticorruption. Les personnes incriminées par cette dernière peuvent, en effet, conserver leur statut officiel tant qu'elles n'entrent pas dans la catégorie des suspects ${ }^{329}$.

Entre temps, le 17 avril, Badrodin Haiti est officiellement nommé chef de la police indonésienne par Jokowi ${ }^{330}$, et Budi Gunawan est finalement nommé souschef de la police ${ }^{331}$ par Haiti ; néanmoins, la crise continue. Fin avril, le chef du KPK, Abraham Samad, est mis en observation par la police et remplacé temporairement $^{332}$ Il apparaît alors que seule une personnalité forte et consensuelle, capable de ménager l'équilibre des forces entre le gouvernement, la police et la justice peut permettre à la Commission de reprendre pied. Pour choisir un chef au KPK, Jokowi constitue alors un comité qui effectue, début septembre, une présélection de huit candidats ${ }^{333}$.

À ce problème ponctuel, vient s'ajouter celui qui a trait à la situation économique du pays et plus particulièrement au recul de la monnaie nationale et à la baisse relative du taux de croissance.

\section{Compromis face à la critique économique}

Dès la mi-décembre 2014, la roupie atteint son niveau le plus bas depuis $2008^{334}$ et malgré les mesures que prend le gouvernement - création du Service de coordination d'investissement, qui solidarise les différents ministères pour favoriser l'afflux de capitaux étrangers ${ }^{335}$, appui soutenu aux exportations, avantage aux transactions internes en roupies et non en dollars -, elle reste faible et le taux de développement économique relativement bas $(4,7 \%)^{336}$. De ce fait, à la

${ }^{329}$ "Percaya Panitia Seleksi Pimpinan KPK », Tempo, vol. 14, 7 juin 2015, p. 10 ; Taufik Alwie $e t$ d., "Pertaruhan di Sidang Praperadilan », Catra, vol. 31, 10 juin 2015, p. 93-95.

330 « Dilantik Jokowi, Badrodin Haiti Resmi Jadi Kapolri », Kompas.com, 17 avil 2015, (http://nasional.kompas.com/read/2015/ 04/17/09274001/Dilantik.Jokowi.Badrodin.Haiti.Resmi.jadi.Kapolri).

331 "Penobatan yang Mendadak », Tempo, vol. 9,3 mai 2015, p. 28

332 "KPK Sekarang lbarat Mobil Mogok », Tempo, val. 52, ker mars 2015, p. 39. La crise se double de tentatives d'intimidation effectuées au domicile de certains membres du KPK à partir du mois de juillet, "Teror untuk penyidik KPK ", Tempo, vol. 21, 26 juillet 2015, p. 30-31.

${ }_{333}$ Wayan Agus Purnomo et Aan Pranata, "Karpet Merah Kandidat Kontroversial », Tempo, vol, 28, 13 septembre 2015, p. 41 43.

334 "Kaleidoskop 2014: Rupiah Tersungkur ", Tempo, vol. 44, 4 janvier 2015, p. 69.

335 "Baru 100 Hari Pertama ", Tempo, vol. 49, 8 février 2015, p. 26.

336 "Terapi Atasi Permasalahan Rupiah », Tempo, vot. 3, 22 mars 2015, p. 92. 
mi-mai, des proches du président -- dont Megawati Soekarnoputri, chef du PDI-P et ancienne présidente d'Indonésie, et Jusuf Kalla - font pression sur Jokowi pour qu'il remanie le gouvernement ${ }^{337}$. Jokowi attend le 12 août pour finalement renouveler 6 de ses ministres. En lien avec la situation économique, il change le ministre coordinateur des Affaires économiques et le ministre du Commerce, ainsi que le chef du Bureau national du planning de développement, le Bappenas $^{338}$.

Face à ce changement lié au quotidien d'un gouvernement, des réformes plus profondes sont lancées, qui pourraient - si elles sont menées à terme - améliorer significativement la situation économique et sociopolitique de l'Indonésie.

\section{Le mouvement de réformes}

\section{Gestion des ressources naturelles}

\section{Pertamina, Petral ef l'exploitation du pétrole}

L'entreprise d'État Pertamina, fondée en 1968 pour gérer la production de pétrole et de gaz du pays, représente l'un des symboles forts de la période suhartoïste (laquelle débute en 1965) et de ses pratiques. Elle est, en effet, réputée receler un condensé de méthodes de collusion et de corruption ${ }^{339}$. Voilée par un subtil et très efficace jeu de prête-noms, de fausses factures, de pots-de-vin, de sociétés fictives dans des paradis fiscaux (Hong Kong, Singapour) et de manipulation d'hommes de paille jusqu'aux plus hautes sphères politiques et économiques, l'action ainsi menée dans l'ombre permettrait de détourner des fortunes

\footnotetext{
${ }^{337}$ Sunudyantoro, " Dalam Tekanan Delapan Penjuru Mata Angin ", Tempo,vol. 12, 24 mai 2015, p. 33-41.

${ }^{338}$ " jokowi inaugurates six new ministers », jakarta Post, 12 août 2015, (www.thejakartapost.com/news/2015/08/12/jokowiinaugurates-six-new-ministers.html).

339 Voir, par exemple, Tempo,co, 21 octobre 2008, (http://www.tempo.co/read/fokus/2008/10/21/229/Bos-Cold-Manor-jadiTersangka-Kasus-Zatapi.).
} 
colossales pour bénéficier au profit personnel ou bien pour être réinvesties dans le financement des campagnes électorales ${ }^{340}$.

Peu de personnes s'y retrouvent dans cet imbroglio, il est néanmoins notoire que Petral, sous-groupe en charge de gérer les échanges commerciaux de la Pertamina, est au cœur de ces pratiques, au point que des observateurs avertis estiment que c'est Petral qui - depuis Singapour - dirige en réalité la Pertami$\mathrm{na}^{341}$. De fait, l'attention du ministre de l'Énergie et des Ressources naturelles de Jokowi, Sudirman Said, se porte sur cette société. Pour ce faire, il constitue en novembre 2014 un groupe chargé d'étudier les processus d'autorisations et d'échanges économiques de pétrole et de gaz naturel au sein des organismes d'État et de donner des recommandations ${ }^{342}$.

Ensuite, au mois d'avril 2015, la ministre des Entreprises d'État (Badan Usaha Milik Negara, BUMN), Rini Soemarno, déclare son intention de liquider Petral. En mai, on annonce que la liquidation aura lieu après l'audit financier de Petral qui durera jusqu'en avril $2016^{343}$. La question demeure de savoir si cette liquidation aura un effet sur les pratiques de collusion de la Pertamina.

\section{Exploitation minière et bras de fer avec Freeport}

Les relations avec les sociétés en charge de la gestion des produits miniers apparaissent, comparativement au cas d'étude précédent, bien plus limpides. L'action du gouvernement Jokowi en la matière s'inscrit dans la continuité de l'interdiction d'exporter des minerais bruts. Un bras de fer s'engage ainsi entre le gouvernement indonésien et les sociétés étrangères en cours de contrat dans le pays. Le cas de la société américaine Freeport est représentatif de ce type de rapport, de même qu'il est aussi évocateur des relations ambiguës que le régime suhartoïste entretenait avec les sociétés occidentales : un développement qui profite avant tout à celles-ci et au régime en place $^{344}$.

${ }^{340}$ " "Siapa Sih Mafia Migas Itu ?"Kesaksian Pelaku (1)", Baranews.co, 9 août 2014, (http://baranews.co/web/ read/19024/siapa.sih.mafia.migas.itu.kesaksian.pelaku.1\#.VkKWS-LYHvO); Komisi Kepolisian Indonesia, 25 août 2013. (http://www.komisikepolisianindonesia.com/umum/read/12745/mafia-migas-indonesia.html).

${ }^{341} /$ bid., cf. note 29.

342 "Kaleidoskop 2014: Pembentukan Tim Reformasi Migas », Tempo, vol. 44, 4 janvier 2015, p. 70.

${ }^{343}$ Ayu Prima Sandi et al., "Satu Kemudi Jual-Beli Minyak », Tempo, vol. 13, 31 mai 2015, p. 130-132.

${ }^{344}$ L'extraction minière effectuée par Freeport se situe, de plus, en Papouasie occidentale, lieu de nombreux massacres perpétrés par l'armée durant ce régime. Depuis la chute de celui-ci en 1998, la région est restée interdite d'accès et des meurtres ont 
Le gouvernement doit donc parvenir à imposer sa réglementation, datant de 2009, d'obligation de traitement des produits bruts sur le sol indonésien. Mais il doit aussi ménager les entreprises étrangères. À titre d'exemple, l'extraction effectuée par Freeport en Papouasie occidentale occupe 30000 personnes (troisième mine de cuivre et deuxième pour l'or au rang mondial) ${ }^{345}$. Finalement, Freeport finit par accepter d'investir 2,3 milliards \$ US pour financer un site de raffinement à Gresik et envoie en juin des cadres de la société pour discuter du contenu des autres points (réduction du périmètre d'exploitation, durée du contrat, augmentation du pourcentage versé à l'État indonésien, augmentation du personnel local).

\section{Les « combats navals » de la ministre de la Mer}

Le domaine maritime indonésien est emblématique du pays puisqu'on y retrouve l'idée d'archipel dans les deux noms principaux que porte celui-ci : le nom officiel, Indonésie (« les îles indiennes »), et le nom de la tradition javanaise, Nusantara («les îles extérieures»). Il n’est donc pas étonnant que le premier président, Sukarno, ait eu des ambitions maritimes très marquées qui se sont exprimées au travers de la constitution de la flotte militaire indonésienne - une des plus importantes du monde au début des années 1960 - avant qu'elle ne soit désarmée par Suharto, général de l'armée de terre qui a supplanté Sukarno en 1965. Depuis cette époque, les eaux indonésiennes ont perdu de leur prestige, notamment victimes ici aussi de pratiques de corruption et de collusion, sur fond d'exploitation sauvage de l'environnement marin.

Pour assumer l'ambition qu'il a de réhabiliter ce domaine, le choix du ministre de la Mer par Jokowi se porte sur Susi Pudjiastuti, une femme d'action issue du monde de l'entreprise. Dès le début de son mandat, elle met à jour la réglementation pour lutter contre la pêche illégale dans les eaux territoriales indonésiennes. Très rapidement, elle mène de façon suivie des opérations

continué à s'y perpétrer (Le Monde Asie-Pacifique, 29 janvier 2011, (http://www.lemonde.fr/asie-pacifique/article/ 2011/01/ 29/les-activistes-papous-d-indonesie-se-battent-pour-leur-autodetermination_1472302_3216.html). À cet égard, le président Jokowi prend des mesures très fortes: dès le mois de mai 2015, il autorise l'accès de la région aux journalistes - Kontan, 10 mai 2015 - (http://nasional.kontan.co.id/news/mulai-hari-ini-wartawan-asing-bebas-ke-papua), et libère cinq prisonniers politiques papous - Kontan, 9 mai 2015, (http://nasional.kontan.co.id/news/presiden-jokowi-segera-bebaskan-5-tapolpapua/2015/05/09).

${ }^{345}$ Ismar Patrizki, « Freeport Tak Akan Melanggar Aturan », Tempo, vol. 49, 8 février 2015, p. 89-90. 
d'envergure pour arrêter les contrevenants et couler leurs navires de pêche (philippins, malaisiens, thailandais, chinois, japonais et vietnamiens).

Selon la ministre, ce ne serait pas moins de 3000 trillions de roupies ${ }^{346}$ qui seraient ainsi pillés chaque année, soit 1,5 fois le budget de l'État en $2014^{347}$. Globalement, le volume indonésien de la pêche traditionnelle a effectivement augmenté depuis l'instauration des interdictions pour passer de 3,81 millions de tonnes en 2014 à 4,36 millions de tonnes dès le mois de juin 2015, avec un bénéfice passant de 42,41 trillions de roupies à 50,32 trillions de roupies ${ }^{348}$. Pour intensifier cet élan, le gouvernement lance en 2015 un programme visant à investir 100 milliards de roupies dans chacune des 92 îles les plus périphériques de l'archipel. Il s'agit à la fois d'aider les populations géographiquement excentrées, mais aussi de marquer la présence indonésienne dans ses confins maritimes $^{349}$.

À cette volonté de gestion raisonnée des ressources halieutiques (il est aussi prévu de créer 700 zones maritimes protégées ${ }^{350}$ ) s'adjoint celle - plus difficile à mettre en place - du patrimoine forestier.

\section{Destruction de l'environnement naturel}

Malgré les plaintes des pays voisins - la Malaisie et Singapour - et celles des habitants des régions concernées, les incendies criminels des forêts de Sumatra et de Kalimantan - qui connaissent un pic depuis les années 1990 - continuent d'être perpétrés sans qu'aucune mesure politique sérieuse ne soit prise. Cette année, le bilan reste des plus alarmants : 340 points de feu (303 à Kalimantan et 37 à Sumatra) sont repérés pour 33977 hectares de forêt brûlée, et 122501 personnes se retrouvent victimes d'infections respiratoires souvent mortelles ${ }^{351}$.

\footnotetext{
${ }^{346}$ II faut environ 15000 roupies pour faire un euro.

${ }^{347}$ "Mengawal Gerakan Menteri Susi », Tempo, vol. 40, 7 détembre 2014, p. 31.

${ }^{348}$ Devi Ernis, "Lobi Pengusaha lkan di Meja Istana ", Tempo, vol, 14, 7 juin 2015, p 88-93.

349 "Menteri Susi Gelontorkan $\mathrm{Rp} 100$ Miliar untuk Pulau-pulau Terluar », Kompascom, 20 août 2015 (http://bisniskeuangan.kompas.com/read/2015/08/20/113500826/Menteri.Susi.Celontorkan.Rp.100.Miliar. untuk.Pulau-pulau. Terluar).

350 "Rencana Pembangunan Jangka Menengah Nasional », Tempo, vol. 6, 12 avril 2015, p. 39.

351 " Hutan yang menangis", Tempo, vol. 30, 27 septembre 2015, p. 12. La situation suscite un déplacement spécial du président jokowi fin septembre. A titre d'illustration, le directeur du lycée SMA6 sur lequel j'effectue mes enquêtes prononce le
} 
Les efforts locaux semblent vains pour mettre fin à ces pratiques. Cette fois-ci pourtant, la ministre de l'Environnement et de la Forêt, Siti Nurbaya Bakar, affirme qu'il y a une volonté politique du gouvernement pour changer la situation $^{352}$. Les autorités préfectorales locales refusant de coopérer, de même que les entreprises papetières et de culture du palmier à huile sur lesquelles pèsent tous les soupçons, la politique de Siti Nurbaya vise à contraindre ces compagnies à se responsabiliser elles-mêmes. Si des incendies se développent sur les terres qu'elles exploitent, des sanctions seront prises qui peuvent aller jusqu'au retrait définitif de leur licence ${ }^{353}$.

Enfin, la destruction irraisonnée du plus important patrimoine naturel $\mathrm{du}$ pays, avec la mer, s'accompagne du pillage et du massacre systématique de sa faune. Si des arrestations ont parfois lieu ${ }^{354}$, la police peine à juguler le trafic d'ivoire dans la région d'Aceh, d'autant que d'anciens guérilleros du Mouvement d'Aceh libre, des membres de l'armée et même de la police, semblent être compromis ; l'éléphant et le tigre de Sumatra apparaissent, de ce fait, être voués à une disparition prochaine ${ }^{355}$. Dans le même ordre d'idées, le gouvernement prévoit qu'après 2015, il ne sera plus possible de remettre en liberté les orangsoutans qui ont grandi en captivité, par manque de forêt pour les accueillir ${ }^{356}$.

\section{Développement social et éducation}

Pour appuyer sa politique de développement social, le gouvernement inscrit dans ses priorités le «Programme familial productif » qui comprend trois volets : aider les familles ayant un besoin économique très marqué, permettre aux enfants de ces familles de pouvoir faire des études, offrir un accès gratuit aux

12 octobre un discours centré sur ce problème, plaignant les habitants qui restent depuis des mois, sans pouvoir voir le soleil du fait des fumées d'incendie. Une quête pour leur venir en aide est organisée le lendemain par les lycéens.

352 " Licensing is not followed by controls », Tempo English, 27 septembre 2015, p. 28.

353 Ibid., p. 26-29.

${ }^{354}$ Yuliawati et Sigit Zulmunir, « Bursa Hewan Langka di Jagat Maya », Tempo, vol. 1, 8 mars 2015, p. 80-81.

355 Ibid. Voir aussi « Tak Ada Gading di Kepala Yinphiek », Tempo, vol. 3, 3 mai 2015, p. 52-61.

${ }^{356}$ Erwin Zachri, " Jalan Pulang yang Panjang ", Tempo, vol. 12, 24 mai 2015, p. 80-81. Une thématique liée est celle de la spoliation des droits fonciers des peuples coutumiers. À ce sujet, la Commission des droits de l'Homme tient pour la première fois à Ambon, aux Moluques, une réunion concernant les conflits fonciers liés aux droits locaux en vue de sensibiliser le nouveau gouvernement à cette question (Tempo English, 16 novembre 2014, p. 50-51). Toujours à propos des peuples minoritaires, une commission d'experts du Conseil de représentation régional () établit le fait que 14 langues ont disparu dans l'année écoulée et que 139 autres sont en voie d'extinction (Tempo, 28 juin 2015, p. 28). 
soins de santé. Du fait d'une trop grande précipitation, le lancement de ce programme est cependant marqué par quelques couacs. En effet, le gouvernement voit dans ces mesures la possibilité de contrebalancer le mécontentement face à l'augmentation du prix de l'essence du fait de l'arrêt des subventions aux produits pétroliers de consommation courante ${ }^{357}$.

En parallèle, le ministre de la Culture et de l'Enseignement primaire et secondaire, Anies Baswedan, entreprend des réformes de fond en vue de réhabiliter le plaisir d'enseigner et d'apprendre - cher au fondateur de l'éducation indonésienne, Ki Hadjar Dewantara, un modèle pour le ministre -, tout en maintenant le caractère compétitif marqué du système éducatif indonésien. Dès sa prise de fonction, il stoppe ainsi la mise en place du programme éducatif de 2013 qu'il juge inadapté. De plus, considérant qu'un seul examen ne peut sanctionner toute une scolarité, il décide que les examens nationaux des collèges et des lycées ne serviront plus à rentrer au lycée ou dans les universités.

Il initie aussi un processus de révision des manuels d'éducation de la religion musulmane et d'éthique de la classe de première du lycée. On y trouve, par exemple, un passage qui dit qu'appeler le nom des prophètes, des cheikhs ou des anges comme intermédiaires - pratique très courante dans le monde musulman est considéré comme l'action de vénérer plusieurs dieux, sans plus de contextualisation que cela. Jugeant ces contenus intolérants, le ministre fait procéder à une enquête suite à laquelle le chef du Bureau des programmes et de publications du ministère de l'Éducation, Ramon Mohandas, constitue une équipe indépendante chargée de vérifier le contenu des livres de classe avant leur diffusion ${ }^{358}$. À partir de mai 2015, Baswedan lance le programme des «Enseignants en première ligne » suivant lequel, en quatre mois, 798 professeurs partent de leur plein gré enseigner dans les zones rurales de 28 préfectures manquant de personnel enseignant. Il espère atteindre le chiffre de 3500 pour l'année prochaine ${ }^{359}$.

357 "Crusa-Grusu kartu Sakti », Tempo, vol, 38, 23 novembre 2014, p. 32 et p. 37-43.

${ }^{358}$ G.A. Hayati Nupus Curitno, « Jejak Intoleransi Buku Ajar », Gatra, vol. 22, 8 avil 2015, p. 34-35.

${ }^{359}$ « Don't ever undermine the value of teachers by monetizing their profession », Tempo Eng/ish, 27 septembre 2015, p. 7. 


\section{Entrée de Jokowi sur la scène politique régionale et internationale}

\section{L'échéance Asean, I'APEC et la Conférence de Bandung}

Durant les premières semaines de son mandat, la volonté de mettre en œuvre au plus vite les réformes sociales et institutionnelles les plus pressantes empêche le président indonésien d'assurer les obligations qui sont les siennes à l'international. Néanmoins, dès le mois de novembre 2014, il ne peut plus contourner cet agenda politique. Outre la participation de Jokowi au G20 (à Brisbane du 15 au 16 novembre $^{360}$ ), les rendez-vous internationaux les plus urgents, au regard des alliances qui se nouent dans la région Asie-Pacifique, sont ceux de l'APEC (AsiaPacific Economic Cooperation) et de l'Asean.

L'APEC se tient à Beijing du 10 au 12 novembre 2014. C'est à cette occasion que Jokowi se présente pour la première fois dans une manifestation internationale. Il en profite pour effectuer des rencontres bilatérales, avec Barack Obama - avec qui il communique aussi en indonésien, langue connue du président américain - sur des questions générales comme celle du terrorisme, et avec son hôte, le président chinois Xi Jinping, qui lui fait des propositions de collaboration dans les domaines de la construction portuaire, ferroviaire et de l'énergie électrique.

Dans le prolongement de l'APEC, la $25^{\mathrm{e}}$ conférence de l'Asean se tient en Birmanie, les 13 et 14 novembre 2014, pour passer à cette occasion sous la présidence de la Malaisie. Les dossiers alors traités sont repris dans les mois suivants par les membres du gouvernement indonésien, notamment par le ministre du Commerce, Rachmat Gobel. Cela comprend la carte bancaire commerciale Asean, la facilitation des activités commerciales au sein de l'Asean, le développement des négociations concernant le RCEP (Regional Comprehensive Economic Partnership) et la mise au point de la zone de marché ouvert au sein des pays d'Asie du Sud-Est. Sur ce dernier point, selon Gobel, les membres de l'Asean se

${ }^{360}$ « Bukan Agenda Pringas-Pringis », Tempo, vol. 34, 26 octobre 2015, p. 46. 


\section{L'ASIE DU SUO-EST 2016}

sont entendus pour fixer comme objectif d'atteindre un développement économique de $5,1 \%$ dès la première année ${ }^{361}$.

À l'issue de la $26^{\mathrm{e}}$ rencontre Asean, à Kuala Lumpur, le président Jokowi, quant à lui, se déclare optimiste à propos de l'aptitude indonésienne à s'adapter à l'ouverture du marché économique dès le début de l'année 2016, grâce à la grande variété des productions du pays ${ }^{362}$. Outre les questions économiques, l'Indonésie est aussi intéressée par les dossiers qui la touchent de près comme - l'institutionnalisation de la résilience liée aux catastrophes ou bien le renforcement de la lutte contre les méfaits de l'islam radical ${ }^{363}$. Nonobstant, un dossier qui ne semble guère avancer est celui des accords sur les eaux territoriales et, plus spécialement, entre la Chine et l'Indonésie.

À ce sujet, les accords liés à l'ouverture des frontières de l'ASEAN Economic Community (AEC) en articulation avec celui qui concerne la zone élargie de marché ouvert du Regional Comprehensive Economic Partnership (RCEP) regroupant l'Asean, la Chine, le Japon, la Corée du Sud, l'Inde, l'Australie et la Nouvelle-Zélande ${ }^{364}$ devraient, à terme, déboucher sur un consensus concernant le partage des domaines maritimes.

Cependant, comme le souligne Bruno Hellendorf ${ }^{365}$, l'important rôle de médiateur qu'a joué l'Indonésie entre le Vietnam, la Chine et les Philippines depuis les années 1990, à propos des eaux territoriales, sera mis en cause lorsque la question des frontières maritimes entre l'Indonésie et la Chine se posera. Le renforcement du budget de la marine va dans le sens d'un positionnement indo-

361 «Pertumbuhan Ekonomi ASEAN Dipatok Lebih Besar dari Ekonomi Clobal», Kompas,com, 4 mars 2015, (http://bisniskeuangan.kompas.com/read/2015/03/04/063800626/Pertunbuhan.Ekonomi.ASEAN.Dipatok.Lebih.Besar.dari.E konomi.Global).

362 "Presiden minta identifikasi produk berdaya saing hadapi MEA 2016", Antara news, 27 avril 2015, (http://www.antaranews.com/berita/493012/presiden-minta-identifikasi-produk-berdaya-saing-hadapi-mea-2016).

Nonobstant ce positivisme ambiant, l'ouverture des frontières économiques intra-Asean mobilise assez peu l'attention de la population indonésienne et des médias, ce qui conduit certains observateurs à formuler des réserves quant au bon déroulement de cette ouverture (Shofwan Al Banna Choiruzzad, 2015, Asean di Persimpangan Sejarah. Politik global, Demokrasi, dan Integrasi Ekonomi, Jakarta, Buku Obor, p. 89-94).

363 "What did the 26th Asean Summit achieve », The Diplomat, 28 avril 2015, (http://thediplomat.com/2015/04/what-didthe-26th-asean-summit-achieve/).

364 «Malaysia as ASEAN chair in 2015. What to expect », The Oiplomat, 22 novembre 2014, (http://thediplomat com/2014/11/ malaysia-as-asean-chair-in-2015-what-to-expect/).

365 Bruno Hellendorf, Enjeux d'une présidence Jokowi. Réformes et relations civilo-militaires en Indonésie, Note d'analyse, Bruxelles, GRIP, 31 actobre 2014, p. 14-15, (http://www.grip.org/sites/grip.org/files/NOTES_ANALYSE/2014/NA_2014-1031_FR_B-HELLENDORFF.pdf). 
nésien ferme. Comme on l'a vu, certains aspects de la politique de la ministre de la Mer vont aussi dans ce sens.

En dehors de la zone Asie-Pacifique, un événement moins médiatisé des activités internationales indonésiennes est lié à la conférence afro-asiatique de Bandung, laquelle est à l'origine du Mouvement des non-alignés. Cette conférence commémore ses 60 ans du 22 au 24 avril 2015 à Jakarta et à Bandung avec vingt et un pays représentés. En ouverture de conférence, le président Joko Widodo fait un discours remarqué dans lequel il déclare que les problèmes économiques ne peuvent plus être résolus par la Banque mondiale, le Fonds monétaire international $^{366}$ ou la Banque de développement asiatique. Il profite par ailleurs de cette occasion pour relancer les échanges économiques et diploma-tiques avec l'Afrique ${ }^{367}$.

\section{Droits de l'Homme}

\section{L'affaire « Bali 9 »}

L'attention se porte beaucoup en 2015 sur les condamnations à mort, décidées par la justice indonésienne, du fait du nombre important de ressortissants étrangers accusés de trafic de drogue. Une première vague de six exécutions a lieu mijanvier incluant deux étrangers. Une autre vague se prépare au mois d'avril qui compte - en plus d'un Indonésien - huit étrangers : deux Australiens, un Brésilien, un Espagnol, un Français, un Ghanéen, deux Nigériens et une Philippine.

Les efforts déployés par les différents gouvernements, par la Ligue des droits de l'Homme, par les associations et par l'opinion publique internationale ne parviennent pas à influencer le président Jokowi pour qu'il accorde sa grâce $^{368}$. L'ancien chef de l'État, Susilo Bambang Yudhoyono, l'avait utilisée dans

\footnotetext{
${ }^{366}$ || reçoit malgré tout la présidente du PMI, Christine Lagarde, à Jakarta début septembre. Celle-ci le félicite d'avoir réduit les subventions à l'essence et de consacrer le budget de l'État au développement infrastructurel (construction de 10 nouveaux ports, $2000 \mathrm{~km}$ d'autoroutes, 10 sites industriels, 9 aéroports de marchandises, locaux pour 5000 marchés traditionnels; Tempo, 12 avril 2015, p. 39), et lui conseille de renforcer l'équilibre fiscal, la monnaie nationale et la valeur d'échange ( IMF minta Jokowi perkuat nilai tukar », Tempo, 13 septembre 2015, p. 101).

367 "Poros Ekonomi Ala Jokowi », Tempo, vol. 3, 3 mai 2015, p. 112.

368 « Dukungan Untuk Hukuman Mati », Tempo, vol. 49, 8 février 2015, p. 10 ; " Camang Mengeksekusi Duo Bali Nine », Gatra, vol. 22, 8 avril 2015, p. 94-102.
} 
un cas de figure similaire, ce qui avait contribué à le rendre impopulaire ; l'Indonésie se montre sensible au fait qu'on respecte sa souveraineté juridique.

La principale raison mise en avant réside dans l'ampleur de la consommation locale de stupéfiants chez les jeunes. Selon les statistiques, 33 personnes meurent chaque jour dans l'archipel du fait de leur utilisation, soit plus de 12000 par $a^{369}$. À ceci, il faut ajouter que l'Indonésie représente le plus important marché de méthamphétamine d'Asie du Sud-Est, soit 219 tonnes pour 2014. On peut aussi constater que les Indonésiens sont traités de la même façon à l'étranger. Ainsi, 131 Indonésiens encourent la peine capitale pour des raisons similaires: 112 en Malaisie, 15 en Chine, 2 au Laos, 1 à Singapour et 1 au Vietnam ${ }^{370}$.

\section{Les réfugiés Rohingya}

Un autre sujet qui met en jeu droits de l'Homme, relations interétatiques et qui reste brûlant cette année est celui des réfugiés Rohingya qui fuient la Birmanie, par terre et par mer, pour rejoindre les pays proches. Le gouvernement indonésien continue d'accorder son aide à ces réfugiés, mais le nombre des bateaux qui restent en mer reste très important (plusieurs milliers). Depuis les côtes birmanes, les réfugiés qui arrivent par voie maritime se rendent en Malaisie (20 000 en 2013 selon les Nations unies), ou bien se rendent en Thailande d'où ils repartent vers les îles Andaman (Inde), vers le Sri Lanka ou vers l'Indonésie. De ce fait, du côté indonésien, on attend de l'Asean la mise au point d'une procédure claire pour régler le problème ${ }^{371}$.

Le profil atypique de Jokowi et l'ampleur des réformes qu'il entreprend confèrent à l'Indonésie une aura particulière au sein de l'Asean - et plus largement de l'Asie-Pacifique -, un peu comme un espoir de réussite d'un modèle démocratique adapté au cas sud-est asiatique, à un moment où la Thailande, la Birmanie, et même la Malaisie, peinént à trouver le modèle sociopolitique qui leur convient.

\footnotetext{
369 "Banyak Jalan ke Nusantara », Tempo, vol. 16, 21 juin 2015, p. 60-61.

${ }^{370}$ Julianti, "Serbuk Putih di Buku Jemani », Tempo, vol. 10, 10 mai 2015, p. 83-84.

${ }^{3 / 1}$ Sandika Prihatnala et al, « Jangan Biarkan Mereka Tenggelam », Gatra, vol. 29,27 mai 2015, p. 80-81.
} 
Néanmoins, le gouvernement Jokowi arrive dans un creux de vague économique, relativement faible mais difficile à surmonter. Ses opposants ont ainsi partie facile à brandir cet argument pour critiquer l'action politique à un niveau qui la dépasse bien souvent, l'économique. La nomination de six nouveaux ministres semble pour l'instant avoir calmé les esprits sur ce point.

Il reste que le positionnement du président indonésien, à la croisée d'enjeux nationaux et internationaux, s'annonce complexe. Il lui faut maintenir l'élan des réformes entreprises tout en ménageant les compromis politiques, poursuivre les mesures sociales garantes de sa popularité malgré un budget limité, maintenir et attirer les investisseurs étrangers sans remettre en question les nouvelles règles qui les concernent, ainsi qu'assurer la sécurité et la souveraineté territoriale indonésienne sans froisser les États partenaires voisins.

Enfin, les orientations qui se décideront au niveau de l'Asie-Pacifique et les implications de l'ouverture des frontières économiques de l'Asean seront parmi les principaux rendez-vous de Jokowi à l'international pour 2016. 


\section{Fiche Indonésie}

Capitale : Jakarta

Superficie : $1904569 \mathrm{~km}^{2}$

Population (UNCTAD, est. 2014) : 257800000 hab.

Langue officielle : indonésien

\section{Données politiques}

Nature de l'État : république

Nature du régime : présidentiel

Suffrage : universel (à partir de 17 ans)

Chef de l'État : président Joko Widodo (prise de fonction le 20 octobre 2014)

Vice-président : Jusuf Kalla (élu en 2014)

Ministre des Affaires étrangères : Retno Lestari Marsudi

Ministre de l'Intérieur : Tjahjo Kumolo

Échéances : élections présidentielle et législatives en 2019

Contentieux territoriaux et maritimes :

Chine : les îles Natuna en mer de Chine du Sud (risque latent)

Malaisie : dans la mer des Célèbes autour du plateau continental Ambalat et délimitation des frontières maritimes situées aux larges des îles Sipadan et Ligitan reconnues malaisiennes en 2002 par la Cour internationale de justice

Palaos: contentieux sur l'extension de la Zone économique exclusive (CEE) de l'Indonésie

Singapour : délimitation de la frontière maritime au nord de l'île de Batam (mer de Chine du Sud)

Timor-Leste : règlement définitif de la frontière terrestre (environ $3 \%$ en suspens) et délimitation de l'intégralité de la frontière maritime

\section{Indicateurs démographiques et sociologiques}

Principaux groupes ethniques (2000) : Javanais (40,6\%), Soundanais (15\%), Madourais $(3,3 \%)$, Minangkabau $(2,7 \%)$, Betawi $(2,4 \%)$, Bugis $(2,4 \%)$, Bantenais $(2 \%)$, Banjar $(1,7 \%)$, autres $(29,9 \%)$

Religions (2000): musulmans $(86,1 \%)$, protestants $(5,7 \%)$, catholiques $(3 \%)$, hindous $(1,8 \%)$, bouddhistes $(1 \%)$, autres $(2,4 \%)$ 
Taux de croissance démographique : $1 \%$

Espérance de vie (PNUD, 2012) : 70,8 ans

Indice de fécondité (UNICEF, 2012) : 2,4

Taux de natalité (UNICEF, 2012) : 19,2\%o

Taux de mortalité (UNICEF, 2012) : 6,3\%。

Taux de mortalité infantile (PNUD, 2013) : $31 \%$

Taux d'alphabétisation (> 15 ans) (PNUD, 2013) : 92,8 \%

Taux d'urbanisation (PNUD, 2013) : 52,2\%

Indice de développement humain (PNUD, 2013) : 0,684 (rang 108)

\section{Indicateurs économiques}

Monnaie : rupiah = roupie indonésienne (1 euro $=15290$ rupiah, au 18 octobre 2015)

Balance des paiements courants (UNCTAD, 2013) : - 28, 45 milliards \$ US

Dette extérieure (UNCTAD, 2012) : 172, 327 milliards \$ US

PIB par habitant en parité de pouvoir d'achat courant (FMI, est. 2014) : 5611,85 \$ US

PIB par habitant en dollars courants (FMI, est. 2014) : 4723, 69 \$ US

Taux de croissance du PIB (UNCTAD, 2014) : 5,1\%

Répartition du PIB par secteur d'activité (UNCTAD, 2012): agriculture $(14,44 \%)$, industrie $(46,95 \%)$, services $(38,61 \%)$

Taux d'inflation (FMI, est. 2014) : 4, $9 \%$

Taux de chômage (FMI, est. 2014) : $6 \%$

Taux de population vivant sous le seuil de pauvreté (PNUD, 2012) : 16,2\%

IDE entrant (stocks) (UNCTAD, est. 2013) : 230,344 milliards \$ US

IDE sortant (stocks) (UNCTAD, est. 2013) : 16,07 milliards \$ US

Principaux partenaires commerciaux (UNCTAD, 2014, ordre décroissant) : Japon, Chine, Singapour, États-Unis, Inde

Importations (UNCTAD, 2014) : 178,179 milliards \$ US

Principaux produits importés : machines et équipements, produits chimiques, carburants, denrées alimentaires

Exportations (UNCTAD, 2014) : 176,293 milliards \$ US

Principaux produits exportés : pétrole, gaz, huile de palme, charbon, appareils électriques, contreplaqué, textile, caoutchouc 


\section{Chronologie}

\section{DÉCEMBRE 2014}

50 Aburizal Bakrie est élu président du Golkar.

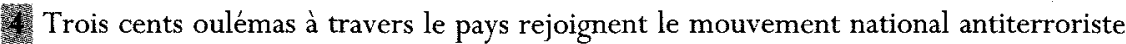
et se déclarent prêts à aider les agences de l'État à détecter les groupes terroristes potentiels.

5. Les autorités maritimes coulent trois bateaux vietnamiens qui pêchaient dans les eaux indonésiennes.

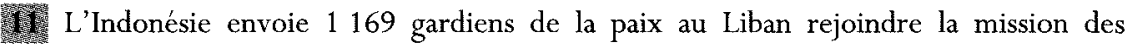
Nations unies, UNFIL.

27 Six hommes suspectés de chercher à rejoindre l'organisation de l'État islamique en Syrie sont arrêtés à l'aéroport Soekarno-Hatta.

28. Un airbus A320 de la flotte AirAsia en route pour Singapour s'abîme au large de Java tuant les 162 passagers et membres de l'équipage à bord.

\section{JANVIER 2015}

16 Après qu'un média a publié la une du journal français Charlie Hebdo représentant Mahomet, le ministre des Affaires religieuses, Lukman Hakim Saifuddin, déclare que les médias sont libres de s'exprimer, mais qu'ils sont tenus de respecter le cadre des règles islamiques.

鹳期 Six individus dont cinq étrangers sont exécutés pour des crimes liés à la drogue, déclenchant une rixe diplomatique avec le Brésil, les Pays-Bas, le Vietnam, le Malawi et le Nigeria.

20 La Chambre des représentants réinstaure officiellement les élections directes pour les élections régionales.

27 La commission pour l'éradication de la corruption (KPK) reçoit des menaces liées à son enquête sur le potentiel futur chef de la police, Budi Gunawan, soupçonné d'avoir détourné 7,5 millions \$ US.

\section{FÉVRIER 2015}

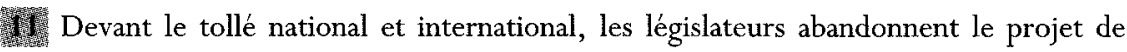
faire passer des tests de virginité aux étudiantes qui obtiennent leur diplôme au lycée. 
12. Le président Jokowi donne l'ordre de faire arrêter toute personne qui chercherait à menacer ou influencer les enquêtes de la KPK après que certains de ses membres ont reçu des menaces de mort.

13 Le secrétaire général des Nations unies, Ban Ki-moon, demande à l'Indonésie de renoncer à la poursuite de l'exécution d'un groupe de prisonniers condamnés pour des crimes liés à la drogue, connu sous le nom de «Bali 9 ».

16. Bien qu'il n'ait pas la capacité juridique de le faire, le juge Sarpin Rizaldi annule le statut de suspect de Budi Gunawan.

18 Le Premier ministre australien Tony Abbot demande à l'Indonésie de montrer de l'indulgence envers les deux Australiens condamnés à mort et de se souvenir du milliard \$ US que l'Australie a donné au pays après le tsunami de 2004.

20 La Cour constitutionnelle révoque une loi de 2004 et met fin au monopole du secteur privé sur l'approvisionnement en eau potable.

22. L'Indonésie menace de revoir son partenariat en matière de défense avec le Brésil, et notamment l'achat d'avions Super Tucano prévu dans le budget 2009-2014, après que le Brésil a rappelé son ambassadeur en signe de protestation contre l'exécution future de son ressortissant, membre du « Bali $9 »$.

24. Une bombe explose dans un centre commercial de Depok sans faire de morts ni de blessés.

27 La police nationale accuse les deux présidents intérimaires de la KPK de plusieurs crimes.

28 Le gouverneur de Jakarta dénonce des irrégularités dans le budget 2015 de la ville, en plus de celles de 2014 repérées par la KPK.

\section{MARS 2015}

5. Un document provenant des archives d'Edward Snowden accuse la Nouvelle-Zélande d'avoir espionné la compagnie indonésienne de télécommunication Telkomsel. Une enquête est ouverte.

9 Seize touristes Indonésiens suspectés d'avoir tenté de rejoindre l'organisation de l'État islamique en Syrie sont arrêtés en Turquie.

18 Le MUI (Indonesian Ulema Council) lance une fatwa condamnant les relations homosexuelles à être punies de coups de canne et, dans certains cas, de la peine de mort.

\section{AVRIL 2015}

18 La France demande à l'Indonésie de ne pas exécuter son ressortissant, Serge Atlaoui, qui fait partie du « Bali $9 »$. 
12. les nations occidentales de piller les ressources de la planète et de s'approprier les richesses produites. Il déclare aussi que le FMI, la Banque mondiale et la Banque asiatique de développement sont incapables de réguler l'économie mondiale.

La police parvient à faire nommer Budi Gunawan au poste de chef adjoint de la police nationale.

14 L'Indonésie exécute huit condamnés à mort dont les deux Australiens et le Brésilien du «Bali $9 »$. L'Australie rappelle son ambassadeur, le Brésil se dit consterné. La Philippine et le Français sont épargnés pour l'instant.

\section{MAI 2015}

㧼 Le président Jokowi donne l'ordre à tous les hôpitaux et, en particulier, ceux du secteur privé, de soigner les détenteurs de la KIS (Indonesian Health Card), sous peine de révocation de leur licence.

La KPK cherche à recruter des militaires pour le poste de secrétaire général afin d'éviter d'autres conflits entre ses enquêteurs et la police. La Chambre des représentants et plusieurs personnalités mettent la KPK en garde car cela risque d'aggraver les tensions entre les institutions et notamment avec la police.

1) Jokowi fait libérer cinq prisonniers politiques papous qui avaient été emprisonnés en 2003. Il déclare également que l'interdiction aux journalistes étrangers d'enquêter dans cette région sera bientôt levée.

17 Jokowi donne une liste de 90 prisonniers politiques enfermés en Papouasie afin qu'ils soient libérés de prison.

19 Le président déclare que 30 bateaux pris en flagrant délit de pêche illégale dans les eaux indonésiennes vont être coulés.

120 L'Indonésie et la Malaisie acceptent d'accueillir 7000 Rohingya prisonniers de bateaux de pêche à la dérive.

2. Le ministre de l'Intérieur Tjahjo Kumolo annonce qu'il n'est plus obligatoire d'indiquer sa religion sur les cartes d'identité.

120 Soixante et un navires de guerre sont déployés afin de sécuriser le territoire maritime à l'ouest de l'Indonésie notamment contre la pêche illégale de bateaux étrangers.

楼) La signature d'un accord de réconciliation entre Aburizal Bakrie et Agung Laksono pour la présidence du Golkar met un terme à des mois de dispute. 


\section{JUIN 2015}

14 Les Nations unies confirment les rumeurs à propos de l'Australie qui aurait payé des pilotes de bateaux de migrants pour qu'ils retournent en Indonésie, enfreignant de ce fait le droit international. L'Indonésie demande des explications à l'Australie.

19 La Malaisie est accusée de pénétrer fréquemment dans les eaux territoriales indonésiennes sans permission. Des bateaux de guerre et des avions de combat indonésiens sont déployés au large de Kalimantan.

22 L'Indonésie déclare qu'en raison du ramadan, l'exécution de Serge Atlaoui est repoussée, bien que celui-ci vienne d'épuiser tous les recours légaux pour défendre son cas. 30 À Sumatra, cent treize personnes meurent dans le crash de leur avion survenu deux minutes après le décollage.

\section{JUILLET 2015}

3 Le ministre des Affaires religieuses, Lukman Hakim Saifuddin, déclare que le mariage homosexuel ne peut être accepté en Indonésie puisque la population et l'État sont fortement religieux.

10. L'éruption du volcan du mont Raung sur lî̀le de Java perturbe gravement le trafic aérien à cause de nuages de cendres. 25000 personnes sont restées bloquées dans les aéroports.

15. L'Indonésie annonce qu'elle limite les exportations bovines vers l'Australie à 50000 têtes pour ce trimestre, soit une baisse de $80 \%$ par rapport au trimestre précédent.

16 La cathédrale de Jakarta ouvre ses portes aux musulmans pour fêter ensemble la fin du ramadan.

21 Le Jakarta Globe publie les résultats d'une enquête qui montre que les investissements étrangers ont triplé depuis l'élection de Jokowi.

22 Les États-Unis classent l'Indonésie à la $25^{\mathrm{e}}$ place sur 54 démocraties en termes de transparence du financement des élections.

28 À Jakarta, le Premier ministre britannique David Cameron annonce son intention de collaborer avec l'Indonésie dans la lutte antiterroriste et dans l'exécution de grands projets d'infrastructures. Jokowi, de son côté, invite Londres à investir davantage en Indonésie et à baisser les taxes sur un certain nombre de produits indonésiens, comme le poisson.

Un séisme de magnitude 7,2 touche l'ouest de la Papouasie. Quatre bâtiments dont un hôpital sont légèrement endommagés et un jeune homme est porté disparu.

30 Joko Widodo reçoit le président turc Tayyip Erdoğan. Ce dernier souligne qu'ils sont tous deux à la tête d'une démocratie musulmane. 


\section{AOÛT 2015}

La fondation Sukarno, présidée par la fille de feu l'ancien président désigne Kim Jongun lauréat du prix pour «la paix, la justice et l'humanité » soulignant son «combat contre l'impérialisme colonialiste ».

19. 2015.

17: Le président Jokowi soutient la mesure défendue par son gouvernement qui pénalise toute insulte à l'égard du président.

17. Jokowi procède à un remaniement de six ministères dont l'important ministère de la Coordination économique pour relancer la croissance, à son plus faible niveau depuis six ans.

19.4 Trois militants supposés de l'organisation de l'État islamique sont arrêtés à Solo. Ils prévoyaient d'attaquer des églises et un poste de police à l'occasion du $70^{\mathrm{e}}$ anniversaire de l'indépendance de l'Indonésie.

15 In avion de la compagnie indonésienne Air Trigana figurant sur la liste noire de l'Union européenne s'écrase en Papouasie tuant ses 54 passagers.

La Gua Maria d'Ambarawa (Java), la plus grande statue de la Vierge Marie au monde, culminant à 42 mètres, fête son $61^{\mathrm{e}}$ anniversaire devant 30000 fidèles. L'évêque du diocèse remercie les autorités locales pour leur engagement en faveur du dialogue interreligieux.

18 8 I Indonésie coule 38 bateaux de pêche illégaux, dont certains proviennent de Chine, du Vietnam, de Thailande et des Philippines.

27 Jokowi encourage la diffusion de davantage de «programmes patriotiques » à la télévision.

\section{SEPTEMBRE 2015}

14. La directrice générale du FMI, Christine Lagarde, et le président Jokowi analysent ensemble à Jakarta les conséquences du ralentissement chinois sur la croissance indonésienne, moins élevée que prévue.

2. Le gouvernement indonésien annonce une forte dérégulation de l'industrie manufacturière, du commerce et de l'agriculture afin de stimuler les investissements.

Le Parti du mandat national rejoint la coalition de soutien à Jokowi.

Le ministère de l'Environnement présente son plan pour réduire de $29 \%$ les émissions de gaz à effet de serre d'ici 2030.

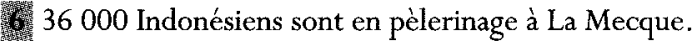


10 L'Indonésie fait son retour dans l'Opep.

Le vice-porte-parole de la Chambre des représentants risque d'être sanctionné pour s'être rendu à un meeting de Donald Trump, candidat à la présidence des États-Unis.

11 Le chef de l'Agence nationale des renseignements intérieurs, Zulkifli Hasan, appelle à une augmentation du budget pour 2016. Il dispose actuellement de 1900 agents mais estime que 5400 sont nécessaires pour garantir la sécurité du pays.

14 État d'urgence à Sumatra après de forts incendies.

15 Mille six cents militaires sont dépêchés à Sumatra pour lutter contre les incendies dont l'origine pourrait être criminelle.

20 Incendies : sept personnes sont interrogées, elles sont suspectées d'avoir allumé des feux afin de défricher de larges terrains pour l'exploitation de palmiers à huile.

25 Un séisme de magnitude 6,6 touche le Nord-Ouest de Sulawesi faisant plus de 60 blessés.

28 Deux journalistes britanniques comparaissent devant la cour de Batam, à l'ouest du pays, pour avoir tenté de faire un reportage sur la piraterie, sans détenir le visa approprié.

30. Jokowi refuse de présenter des excuses officielles pour la répression anticommuniste du 30 septembre 1965 .

\section{OCTOBRE 2015}

5. Les deux factions rivales du Golkar se sont vues pour discuter de l'organisation d'un congrès exceptionnel.

9 L'Indonésie accepte finalement l'aide de Singapour, de la Malaisie, de la Corée du Sud, de la Russie, de l'Australie et de la Chine pour venir à bout des incendies qui frappent Sumatra.

22. Après Singapour et la Malaisie, les fumées des incendies sumatranais touchent désormais le Sud de la Thaillande, perturbant le trafic aérien.

26. Après sa visite officielle aux États-Unis, le président Jokowi annonce avoir conclu pour 20 milliards \$ US de contrats avec des entreprises américaines.

27 Selon un sondage sorti à l'occasion du premier anniversaire de l'élection de Jokowi, l'action de ce dernier est soutenue par 49,2\% de la population.

30 Les fortes pluies ont finalement raison des fumées sumatranaises. 


\section{NOVEMBRE 2015}

8 Un séisme de magnitude 6 a lieu au large de Sumatra, mais il n'y a pas d'alerte au tsunami.

28. Le président Jokowi se rend au Sommet de la COP21 à Paris et y prononce un discours.

\section{DÉCEMBRE 2015}

1.7. Les enquêteurs indonésiens concluent que l'accident de l'Airbus A320 de la flotte AirAsia en décembre 2014 a été causé par une « pièce défectueuse ». 


\section{Portraits}

\section{Susi Pudjiastuti, ministre de la Mer et de la Pêche}

Pudjiastuti est la personnalité politique la plus médiatisée et la plus populaire d'Indonésie après Jokowi. Comme ce dernier, elle vient du monde des affaires où elle se fait remarquer grâce à ses réussites commerciales dans la vente de produits halieutiques, puis dans le transport aérien commercial. Son style direct et enjoué, ses bonnes relations avec le personnel qu'elle emploie et le fait qu'elle n'ait pas terminé ses études au lycée la rendent sympathique aux yeux du public, mais néanmoins antipathique pour certains ${ }^{372}$.

Elle prend très au sérieux la mission que lui assigne Jokowi de défendre le patrimoine marin indonésien. De cette façon, elle perçoit mal la condamnation du capitaine du Hai $\mathrm{Fa}$ - navire qui arbore pavillon panaméen, appréhendé début avril 2015 - à seulement 200 millions de roupies par un juge de la ville d'Ambon, pour naviguer sans autorisation, sans système de positionnement, et avec à bord 900 tonnes de poissons et de crevettes, dont 15 tonnes de requinsmarteaux dont la pêche est interdite ${ }^{373}$. Pour éviter les risques de récidive du fait d'une peine encourue très faible, la ministre en vient donc à opter le plus souvent pour une solution plus radicale. C'est ainsi qu'en début d'année 52 navires étrangers sont envoyés par le fond ${ }^{374}$.

Jusque-là, les navires étrangers parvenaient à opérer en toute impunité en arborant le pavillon indonésien, en utilisant un prête-nom ou encore en pratiquant le trans-shipping (transborder la marchandise d'un navire à l'autre). L'action de Pudjiastuti a aussi une portée qui va au-delà des seules questions territoriales. Par exemple, des capitaines de navires affrétés par la société PT Pusaka Benjina Resources (prête-nom pour une société thailandaise) sont accusés de trafic d'êtres humains (on a trouvé à leur bord des membres d'équipage originaires de Birmanie, du Cambodge et du Laos), de transgression des droits de

\footnotetext{
${ }^{372}$ Mulyawan Karim et. al., 2015, Untold Story Susi Pudjiastuti. Dari Laut ke Udara, Kembali ke Laut, Jakarta, Penerbit Buku Kompas.

${ }^{373}$ Aries Kelana, et al., « Vonis Kontroversial Hai Fa », Gatra, vol. 22, 8 avril, p. 84-85; Tempo, 15 avril 2015, p. 100-101.

${ }^{374}$ Devy Ernis, « Lobi Pengusaha Ikan di Meja Istana », Tempo, vol. 14, 7 juin 2015, p. 88-93.
} 
l'Homme, d'utilisation de faux documents d'immigration, de matériel de pêche non conforme, etc. ${ }^{375}$

\section{Anies Baswedan, ministre de l'Éducation ${ }^{376}$}

Anies Baswedan ne présente pas la même trajectoire que $S$. Pudjiastuti, mais il partage avec elle la volonté d'appliquer une action appropriée, quitte à aller à l'encontre des pratiques en cours. Son parcours du combattant débute tôt puisqu'il occupe des postes à responsabilité en continu depuis le collège en passant par le lycée et jusqu'à l'université. À l'université Gadjah Mada, où il étudie l'économie, il devient en 1992 le président du sénat des étudiants. Il est actif dans les associations éducatives et dans les milieux activistes critiques vis-àvis du régime suhartoïste. Pour sa licence et son master, il obtient des bourses japonaise, puis américaine, qui lui permettent d'étudier dans ces deux pays et d'obtenir un prix de la part de l'Asean. Il termine son doctorat en sciences politiques à l'université d'Illinois (1999) avant de devenir directeur de recherche à The Indonesian Institute (2005, Jakarta). Puis, il devient consultant pour I'ONG internationale Kemitraan financée par le Programme des Nations unies pour le développement. Ce travail lui permet de visiter l'Indonésie jusque dans ses régions les plus reculées et de prendre conscience qu'un potentiel humain remarquable, isolé des préoccupations nationales, ne demande qu'à être valorisé. Il est alors convaincu que le système éducatif national a un rôle à jouer pour y parvenir. En 2007, il devient le plus jeune recteur d'Indonésie à l'université Paramadina (Jakarta). Il crée en 2010 la Fondation du mouvement l'« Indonésie enseigne » qui envoie les meilleurs élèves du pays enseigner pendant un an dans les régions défavorisées. Deux ans plus tard, il lance le mouvement très suivi des «classes d'inspiration»: des professionnels de tous corps de métiers vont enseigner une journée durant dans une école primaire. En 2010, il est aussi sollicité par la Commission de lutte contre la corruption (KPK) pour intégrer une de ses équipes. En 2013, du fait de son intégrité notoire, il est nommé chef du comité d'éthique du KPK.

\footnotetext{
${ }^{375}$ Taufik Alwie et al. « Di. Laut Mereka Diperbudak », Gatra, vol. 24, 22 avril 2015, p. 20-22.

${ }^{376}$ Muhammad Husnil, 2014, Melunasi Janii Kemerdekaan. Biografi Anies Baswedan, Jakarta, Zaman.
} 


\section{Affiche politique}

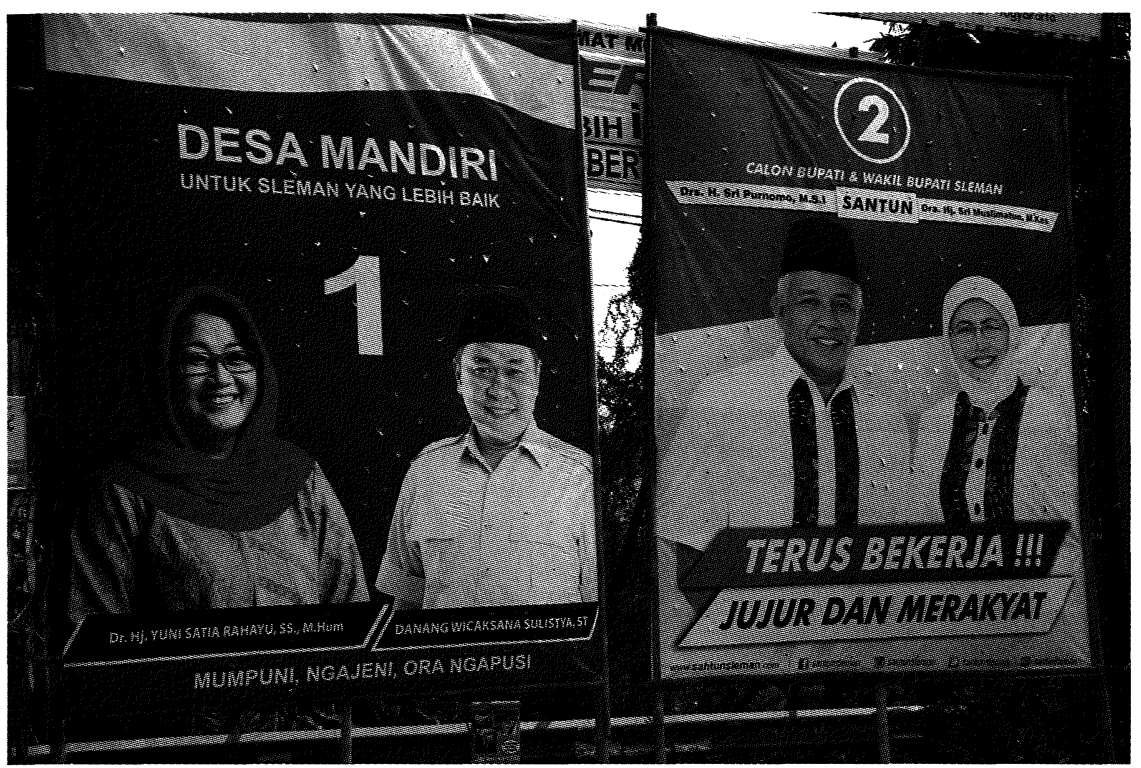

Candidats à l'élection du préfet et de son adjoint pour le district de Sleman (nord du territoire spécial de Yogyakarta) : Yuni Satia Rahayu pour le Partai Demokrat Indonesia Perjuangan (PDIP), Sri Purnomo pour le Partai Amanat Nasional (PAN).

(c) Pascal Dubois. 